\title{
New insights into the regulation of TLR signaling
}

\author{
Sinead M. Miggin ${ }^{1}$ and Luke A. J. O’Neill \\ School of Biochemistry and Immunology, Trinity College Dublin, Ireland
}

\begin{abstract}
Toll-like receptor (TLR) activation is dictated by a number of factors including the ligand itself and the localization of the receptor, in terms of expression profile and subcellular localization and the signal transduction pathway that has been activated. Recent work into TLR signal transduction has revealed complex regulation at a number of different levels including regulation by phosphorylation, targeted degradation, and sequestration of signaling molecules. Here, we describe recent advances that have been made in our understanding of how TLR signaling is regulated at the biochemical level. J. Leukoc. Biol. 80: 220-226; 2006.
\end{abstract}

Key Words: inflammation $\cdot$ regulatory molecules

\section{INTRODUCTION}

Toll-like receptors (TLRs) play a critical role in the evolutionary conserved innate immune response and represent our first line of defense against invading pathogens. TLRs are activated in response to a broad spectrum of pathogen-associated molecular patterns (PAMPs) ranging from bacterial and viral components to fungal and protozoal molecules. The ability to orchestrate an appropriate primary immune response to a vast array of diverse PAMPs is key in our ability to ward off potential pathogenic organisms. The capacity not only to respond appropriately but also to self-regulate host responses to invading pathogens is vital in our ability to mount an appropriate primary immune response.

To date, 11 human TLRs and 13 mouse TLRs have been identified. It has been shown that TLRs are activated by specific PAMPs and that the ability of specific TLRs to heterodimerize adds further to the diverse range of pathogens that may be recognized [1]. For example, TLR1, -2, -4, -6, and -10 recognize lipids. Specifically, lipopolysaccharides (LPS) from Gram-negative bacteria are recognized by TLR4. TLR2, in combination with TLR1 or TLR6, recognizes triacyl lipopeptides and diacyl lipopeptides, respectively. In contrast to the plasma-membrane localization of the aforementioned TLRs, the so-called antiviral TLRs, TLR3, -7, -8, and -9, are endosomally localized, membrane-bound receptors. TLR3 recognizes double-stranded (ds)RNA, which is produced from many viruses during replication. TLR7 recognizes synthetic imidazoquinoline-like molecules, guanosine analogs such as loxoribine, and single-stranded (ss)RNA derived from viruses such as human immunodeficiency virus. TLR8 mediates the recognition of imidazoquinolines and ssRNA. TLR9 recognizes bac- terial and viral CpG DNA motifs. In addition, a third class of TLRs has been defined, sensing protein ligands. Specifically, TLR5 recognizes flagellin, and human TLR11 recognizes uropathogenic Escherichia coli [2]. Recently, the first defined ligand for TLR11 has been described in mice as a profilin-like protein, a class of actin-binding proteins present in apicomplexan protozoa [3, 4]. After recognition of PAMPs, a cascade of intracellular signaling events is activated, which culminates in the induction of proinflammatory cytokines such as tumor necrosis factor $\alpha$ (TNF- $\alpha$ ), interleukin (IL)-6, IL-1 $\beta$, and IL12. In addition, antiviral type I interferons (IFNs; IFN- $\beta$ and multiple IFN- $\alpha$ ) are induced by TLR3, -4, -7, -8, and -9. Moreover, TLRs induce dendritic cell maturation, essential for the induction of pathogen-specific adaptive immune responses [5]. Activation of the intracellular signaling cascades is facilitated by the initial formation of multiprotein complexes containing the TLR and proximal cytoplasmic Toll/IL-1 receptor (IL-1R; TIR)-containing adaptor molecules such as myeloid differentiation primary response protein 88 (MyD88), MyD88 adaptor-like (Mal), also known as TIR domain-containing adaptor protein, TIR domain-containing adaptor-inducing IFN- $\beta$ (Trif), and Trif-related adaptor molecule (TRAM) [6].

Herein, we describe recent advances in our understanding of how the TLR signaling pathway may differentially regulate its response, depending on the nature of the invading pathogenic organisms. Also, we will focus on the inherent ability of our immune system to self-regulate its response to PAMPs to avoid exaggerated responses to pathogens, which may ultimately lead to pathophysiological states.

\section{THE TLR ECTODOMAIN}

All TLRs are type I transmembrane receptors characterized by a highly variable extracellular region containing a leucine-rich repeat domain (LRR) involved in ligand binding and an intracellular tail containing a highly conserved region, the TIR homology domain, which mediates interaction between TLRs and downstream signaling molecules. Although the molecular structure of the TIR domains of TLRl and TLR 2 had been defined [7], it became increasing important to characterize the extracellular LRR with a view to expanding our understanding of how ligands interact with TLRs. Recently, the crystal structure of the TLR3 ectodomain has been solved at $2.1 \AA[8,9]$.

\footnotetext{
${ }^{1}$ Correspondence: School of Biochemistry and Immunology, Trinity College Dublin, Dublin, Ireland. E-mail: miggins@ted.ie

Received November 17, 2005; revised March 21, 2006; accepted March 24, 2006; doi: 10.1189/jlb.1105672.
} 
It is a large, horseshoe-shaped solenoid assembled from 23 LRR stabilized by asparagine residues in the 24-residue LRR motif contributing to hydrogen-bonding networks. The ectodomain is heavily glycosylated with one face on the outer convex surface completely devoid of any glycosylation. This region is the predicted site for dsRNA binding. This is an important finding, as it was previously thought that ligand binding occurred solely at the inner-concave surface. In addition, the nonglycosylated region within the concave surface contains areas of positively charged residues, which are predicted to provide a binding surface for dsRNA and may facilitate TLR3 dimerization. Given the complexity of the ectodomain, it is possible that TLRs may bind different ligands at alterative sites and thus, may impact on the assembly of the intracellular, multiprotein-signaling complex. Thus, future studies will provide a clearer understanding of how TLRs recognize such a diverse array of PAMPs.

Mutations have been reported in the ectodomain of TLR4. For example, Asp299Gly and Thr399Ile polymorphisms in the TLR4 ectodomain predispose to severe malaria [10], possibly by impacting on ligand recognition. This is currently an intense area of research and will expand our broader knowledge into how we have selectively evolved to meet pathogenic challenges.

\section{TLR LOCALIZATION AND ACCESSIBILITY}

Given that all TLRs possess a LRR, a transmembrane region, and a cytoplasmic domain, it was assumed initially that all would be expressed at the cell surface. This has proven not to be the case. Whereas TLR1, $-2,-4,-5$, and -6 are expressed on the cell surface, TLR3, -7 , and -9 are localized to intracellular endosomal compartments. TLR8 appears to be localized primarily, intracellularly with a small portion expressed on the cell surface [11-16]. It is known that whereas the plasma membrane-localized TLRs recognize PAMPs, unique to bacterial and fungal cells, the intracellular TLRs recognize viral and bacterial nucleic acids. It is possible that the intracellular environment may facilitate ligand recognition by the intracellular TLRs, possibly by ligand modification as a consequence of lysosomal acidification, or perhaps the breakdown of late endosomes or lysosomes may serve to release the nucleic acids from the bacteria/virus. Recently, a number of studies have attempted to address the mechanism by which differential subcellular localization is achieved and the biological significance thereof. A recent study has shown that TLR3 is targeted intracellularly by a 23 -amino acid sequence $\left(\mathrm{Glu}^{727}-\mathrm{Asp}^{749}\right)$ present in the linker region between the transmembrane domain and the TIR domain [17]. In contrast, the intracellular localization of TLR7 is achieved by its transmembrane domain. Despite differences in the mechanism by which they are localized, both receptors actually colocalize preferentially near phagosomes containing apoptotic cell particles [17]. Following viral infection, phagocytes take up apoptotic-infected cells, and lysosomal hydrolyases cause release of the virus-derived RNAs from the intracellular compartments, thus facilitating access of the intracellular TLRs to their respective RNA ligands. Although the viral RNA may be recognized by the so-called antiviral TLRs [18], it must be noted that viralderived dsRNA may also be recognized by the cytosolic RNA helicase retinoic acid-inducible gene-I, inducing type I IFN in a TLR-independent manner [19, 20]. More recently, another study has revealed that intracellular localization of TLR9 facilitates access to viral DNA and prevents recognition of selfDNA [21]. It was shown that the transmembrane region of TLR9, but not the linker TIR and tail regions, is responsible for its intracellular localization. Moreover, a chimeric TLR, TLR9N4C, comprised of the ectodomain of TLR9 and transmembrane and cytosolic regions of TLR4, localizes to the plasma membrane. Although the chimeric receptor responds equally to $\mathrm{CpG}$ when compared with TLR9, the chimeric TLR9N4C does not sense viral DNA. This is because the chimeric receptor does not have access to the nucleic acids in the viral particles. Furthermore, the chimeric TLR, relocalized to the plasma membrane, recognizes self-DNA. Taken together, this suggests first that despite both TLRs being functional, viral particles must be degraded to expose the nucleic acids and facilitate signal activation by TLR9 and second, that intracellular localization of TLR9 prevents its activation by self-DNA, as it does not come into contact with self-DNA. Moreover, it has been shown previously that DNase I is secreted into serum to promote degradation of self-DNA, and mice lacking DNase I develop a lupus-like disease $[22,23]$. Adding further to the complexity regarding the interaction of antiviral TLRs with their respective ligands is the recent finding that self-DNA is also recognized by a novel, TLR-independent mechanism, resulting in activation of the innate immune system [24]. Specifically, DNase II is expressed predominantly in macrophages and cleaves the DNA of engulfed apoptotic cells and the nuclei expelled from erythroid precursor cells during erythropoiesis; DNase II-deficient mice have been shown to accumulate DNA in their macrophages with concomitant IFN- $\beta$ production and fetal lethality [24, 25]. It is surprising that it was shown that ablation of TLR3, TLR9, MyD88, or Trif had no effect on the lethality of DNase II-deficient mice, suggesting that the self-DNA is recognized by a TLR-independent mechanism, yet to be determined [24]. From these studies, it is clear that ligand availability and/or receptor compartmentalization are mechanisms by which TLR signaling has evolved to selfregulate, and TLR localization is key to this phenomenon.

Moreover, in an effort to down-regulate host responses to pathogens, TLRs have evolved mechanisms to control or selfregulate its response to pathogens. For example, the TLR itself may be degraded, thereby making it unavailable for subsequent ligand activation, or its expression may be inhibited by anti-inflammatory cytokines. Targeted degradation is achieved via polyubiquitination and subsequent degradation by the $26 \mathrm{~S}$ proteasome. TRIAD3A was a molecule found to bind the cytoplasmic domain of TLR4 and TLR9, but not TLR2, and to promote the ubiquitination and degradation of TLR4 and TLR9, but not TLR2 [26]. Moreover, overexpression of TRIAD3A reduced TLR4- and TLR9-mediated nuclear factor (NF)- $\mathrm{KB}$ activation in response to LPS and $\mathrm{CpG}$ but not pI:C [26]. Anti-inflammatory cytokines also have the ability to downregulate TLR expression. For example, transforming growth factor- $\beta$ (TGF- $\beta$ ) suppresses the expression of TLR4 [27]. 


\section{ADAPTOR MOLECULES}

As outlined above, there are four functional adaptor molecules: MyD88, Mal, Trif, and Tram [6]. Each of these molecule interacts heterophilically with defined TLRs. As shown in Figure 1, MyD88 is the central adaptor molecule interacting with all TLRs except TLR3. Upon ligand activation, MyD88 recruits members of the IRAK family and TRAF6. IRAK then becomes phosphorylated and dissociates from MyD88, which results in TRAF6 being activated, followed by TAK1/TAB1/ TAB2/TAB3 complex formation and activation via K63-linked ubiquitination. Activated TAKl complex then activates the IKK complex consisting of IKK $\alpha$, IKK $\beta$, and IKK $\gamma / \mathrm{NF}-\kappa \mathrm{B}$ essential modulator, which catalyze ІкB phosphorylation. ІкB is then destroyed by the proteasome pathway, allowing NF-кB to translocate into the nuclei, resulting in proinflammatory cytokine production [1]. This is termed the MyD88-dependent pathway. The second adaptor molecule, Mal, may also be involved in this pathway, and it is thought that Mal acts as a bridging adaptor between TLR4, TLR1/2, TLR2/6, and MyD88 [1]. In addition, the Trif-dependent pathway may be activated, as is the case for TLR3 and TLR4. Whereas Tram is thought to act as a bridging adaptor between Trif and TLR4 [28], Trif interacts directly with TLR3 [29]. Activated Trif recruits TBK1 and together with IKKi, mediate IRF3 phosphorylation, dimerization, and nuclear translocation mediating type 1 IFN induction [30, 31]. Trif also interacts with TRAF6 and RIP1, which mediate NF-кB activation [32-34].

Intracellular signaling instigated via the adaptor molecules may be regulated in a number of ways, including protein phosphorylation, degradation, interaction with inhibitory adaptor molecules, and sequesteration. For example, MyD88s inhibits IL-1 and LPS-induced NF-кB activation. Specifically, overexpression of MyD88s favors formation of MyD88s-MyD88 heterodimers over MyD88 homodimers. In the presence of these heterodimers, IRAK1 is recruited via the death domain

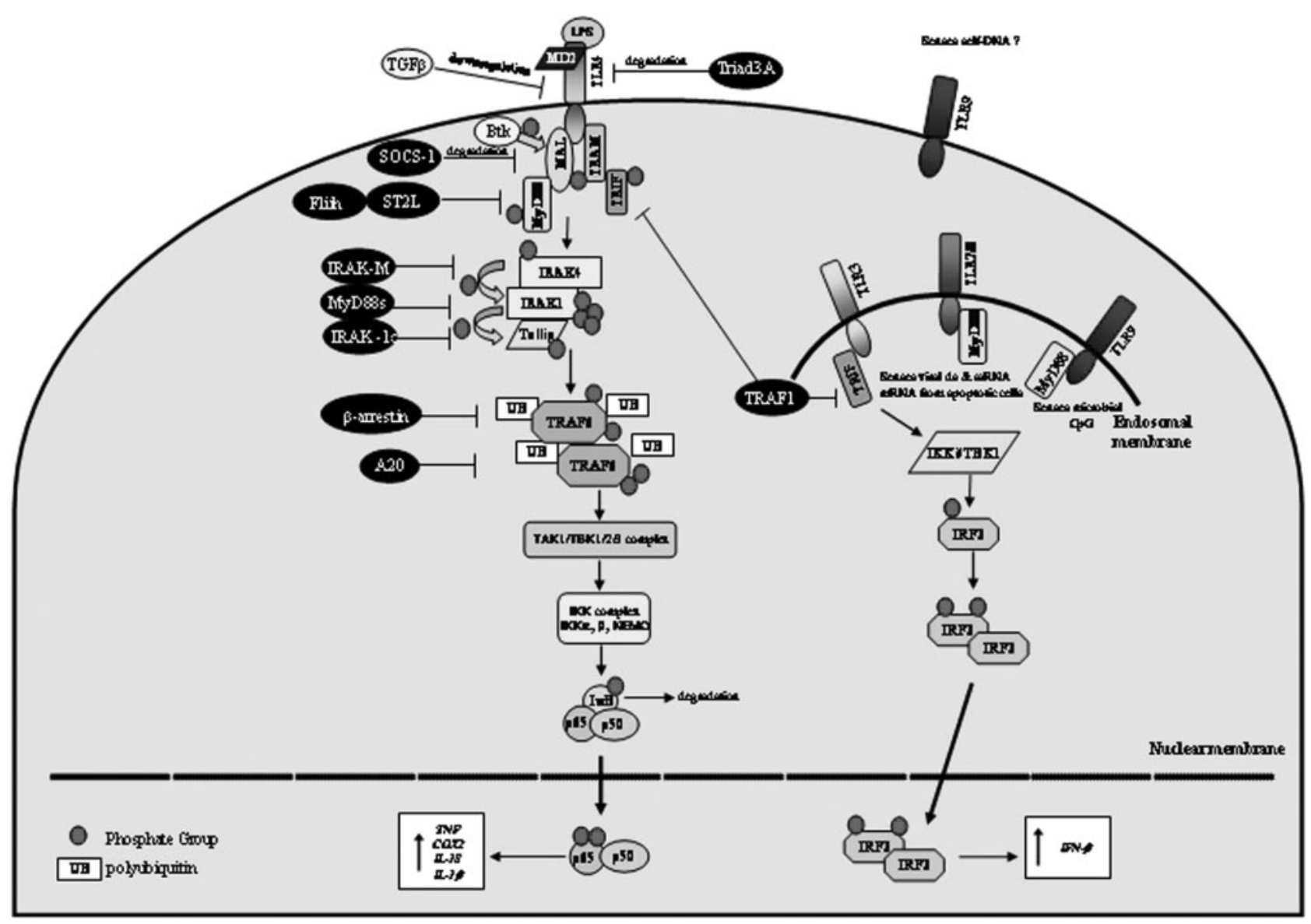

Fig. 1. Regulation of TLR signaling pathways. Whereas TLR4 activates the MyD88-dependent and the Trif-dependent pathways, TLR3 activates the Trif-dependent pathway only. MyD88 recruits IL-1R-associated kinase 4 (IRAK4) and TNF receptor-associated factor 6 (TRAF6) upon ligand stimulation. TRAF6 activates the TGF- $\beta$-activated protein kinase 1 (TAK1)/TAK-1-binding protein-1 (TAB1)/TAB2/TAB3 complex via K63-linked ubiquitination (UB). Activated TAK1 complex then activates the inhibitor of $\kappa \mathrm{B}(\mathrm{I} \kappa \mathrm{B})$ kinase (IKK) complex, which catalyzes IкB phosphorylation and degradation by the proteasome pathway,

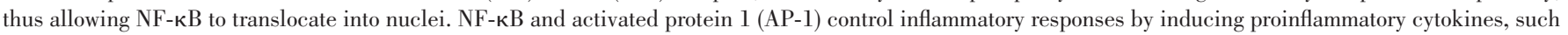
as TNF, IL-1 $\beta$, IL-18, and IL-6. TLR4 also recruits TRAM and Trif, which interacts with Traf family member-associated NF- $\mathrm{B}$ activator-binding protein (TBK1), and TBK1, together with inducible IKK (IKKi), mediates phosphorylation of IFN regulatory factor 3 (IRF3). Phosphorylated IRF3 is dimerized and translocated into the nucleus to bind DNA. Trif also interacts with TRAF6 and receptor-interacting protein kinase 1 (RIP1), which mediate NF- $\mathrm{B}$ activation. Activation of IRF3, NF-кB, and AP-1 is required for induction of type I IFN, particularly IFN- $\beta$. TLR3, which resides in endosomal vesicles, uses Trif but not MyD88, mediating the induction of type 1 IFNs. TLR signaling is negatively regulated by suppressor of cytokine signaling 1 (SOCS-1), Flightless I homologue (Fliih), ST2, Triad3A, Trafl, A20, IRAK-M, IRAK-1c, a short form of MyD88 (MyD88 s), and $\beta$-arrestin, as illustrated. 
(DD), but it is no longer phosphorylated. MyD88s inhibits the ability of IRAK4 to phosphorylate IRAK1, as unlike full-length MyD88, it is unable to interact with IRAK4 [35]. As the kinase is not recruited to the receptor complex, signaling is inhibited. Recently, we have shown that the orphan receptor ST2L negatively regulates IL-1R and TLR4-mediated signaling via adaptor sequesteration [36]. Specifically, we have shown that ST2L, a type I transmembrane protein with three extracellular immunoglobulin-like domains and an intracellular TIR domain, interacts with and sequesters MyD88 and Mal, but not Trif or IRAK. Moreover, a mutant form of ST2L with a mutated proline residue in box 2 of the TIR domain, lost its inhibitory activity. Thus, ST2L inhibits IL-1R and TLR4 signaling by sequesteration of MyD88 and Mal via its TIR domain, thus making them unavailable for signaling [36]. Another molecule, Fliih, has been shown to modulate TLR signaling by interacting with MyD88 and acting as a negative regulator [37]. Fliih contains 16 copies of the LRR motif at its N-terminal region, and the $\mathrm{C}$-terminal regions are homologous to the gelsolin family of actin-binding proteins.

Mal interacts directly with TRAF6 to mediate NF- $\mathrm{kB}$ activation through TLR2 and TLR4 [38] via a putative TRAF6binding motif within Mal. Moreover, mutation of the TRAF6binding motif within Mal, generating MalE190A, inhibited NF- $\mathrm{KB}$ activation, p65-mediated transactivation of gene expression, and $\mathrm{p} 42 / \mathrm{p} 44$ mitogen-activated protein kinase (MAPK) activity. Thus, an intact TRAF6-binding motif within Mal is essential for Mal to signal. It has also been shown that Trif interacts with TRAF6 via a TRAF6-binding motif [34, 39]. Trif was found to mediate TLR3-induced activation of NF-кB via association with TRAF6 [34].

It has also been reported that MyD88, Trif, and TRAM undergo phosphorylation $[34,40,41]$, although the ramifications of phosphorylation remain to be elucidated. Recently, we have shown that Mal is subject to tyrosine phosphorylation [42]. Specifically, Mal may be tyrosine-phosphorylated at positions 86, 106, and 187 during TLR signaling. Moreover, variants of Mal, in which the Tyr 86 or Tyr 187 is substituted with phenylalanine, act as dominant-negative inhibitors of LPS signaling. We identified the Tec kinase, Bruton's tyrosine kinase (Btk), as the kinase responsible for phosphorylating Mal $[42,43]$. These findings suggest that tyrosine phosphorylation is necessary for Mal to signal, although further studies are required to fully explore these possibilities.

More recently, we have shown that SOCS-1 negatively regulates TLR signaling by mediating the degradation of Mal [43]. Specifically, we have shown that after TLR2 and TLR4 stimulation, Mal becomes phosphorylated by Btk and then Mal interacts with SOCS-1, an E3 ligase, mediating Mal polyubiquitination and subsequent degradation via the $26 \mathrm{~S}$ proteasome. Moreover, SOCS-1-deficient mice exhibit potentiation of Maldependent p65 phosphorylation and NF-кB transactivation, leading to amplification of inflammatory signaling [43]. In contrast to the delayed expression of MyD88s and ST2L, targeted degradation of Mal by SOCS-1 represents a more rapid mechanism of regulating TLR signaling.

These studies show that TLR signaling may be regulated by alternative adaptor use, depending on the initial activation signal. Thereafter, the adaptors may be regulated positively and negatively by phosphorylation. Also, targeted degradation following ubiquitination and interaction with negative regulatory molecules may impact on adaptor functionality. In addition, adaptor molecules may be down-regulated by anti-inflammatory cytokines. For example, it has been shown that TGF- $\beta$ induces MyD88 degradation by the proteasome and so downregulates TLR signaling [44]. It is interesting that despite phosphorylated MyD88 being detected in cell lysates following LPS exposure [41], MyD88 is not subject to SOCS-1-mediated degradation.

\section{IRAKs}

Four IRAK family members have been identified and are characterized as having an N-terminal DD to facilitate interaction with MyD88 and a C-terminal kinase domain. Whereas IRAK-1 and IRAK-4 are catalyically active, IRAK-2 and IRAK-M are inactive as a result of an absence of key residues in their respective kinase domains [45-48]. Upon activation of TLR signaling, IRAK-1 and IRAK-4 are recruited to the receptor complex. At the receptor, IRAK-1 associates with Toll-interacting protein (Tollip), MyD88, and TRAF6, and phosphorylation by IRAK-4 triggers IRAK-1 hyperphosphorylation and dissociation of IRAK-1 from the complex while still maintaining its engagement with TRAF6.

IRAKs are subject to regulation at a number of levels including interaction with inhibitory molecules and inhibition of expression. A number of these inhibitory molecules are well defined. For example, IRAK-M is an induced negative regulator that blocks IRAK-4 activation and subsequent IRAK-1 phosphorylation [49]. Tollip itself has been regarded as an inhibitory molecule. As stated above, Tollip interacts with IRAK-1, and IRAK-1 autophosphorylation is suppressed in the presence of Tollip. Following TLR stimulation, IRAK-1 phosphorylates Tollip, and this may lead to the release of Tollip from the Tollip:IRAK-1 complex, thus overcoming negative regulation [50]. More recently, studies have shown that Tollip regulates the magnitude of inflammatory cytokine production in response to IL-1 $\beta$ and LPS [51]. Specifically, Tollip-deficient mice exhibited attenuated responses to the proinflammatory cytokines, IL-6 and TNF- $\alpha$ [51], suggesting that Tollip may function in fine-tuning the response to cytokines.

More recently, an inhibitory splice variant of IRAK-1 was identified [52]. Unlike IRAK-1, the spliced variant IRAK-lc lacks kinase activity and cannot be phosphorylated by IRAK-4 but still retains its ability to interact with IRAK-2, MyD88, Tollip, and TRAF6. Therefore IRAK-lc functions as a dominant-negative by failing to be phosphorylated by IRAK-4 and thus remains associated with Tollip and blocks NF- $\mathrm{KB}$ activation. Also, two splice variants of murine IRAK2, namely IRAK2c and IRAK2d, are inhibitory. Unlike IRAK2, these variants lack a DD and inhibit LPS-induced NF- $\mathrm{KB}$ activation [53]. Moreover, treatment of the mouse macrophage cell line RAW264.7 with LPS for 1 and $3 \mathrm{~h}$ resulted in an increase in IRAK2c mRNA levels, indicating a possible negative-feedback loop [53].

There is another mechanism by which TLR signaling via modulation of IRAKs involves its down-regulation at the 
mRNA level. Specifically, it has been shown that inhibition of IRAK-1 expression at the mRNA and protein levels is the mechanism by which $\mathrm{Pam}_{3} \mathrm{Cys}_{\mathrm{S}} \mathrm{SK}_{4} 3 \mathrm{HCl}$-induced tolerance via TLR2 is mediated [54]. A similar mechanism of decreased IRAK-1 protein was evident following exposure of peritoneal macrophages to the TLR7/8 ligand, R848 [55]. Moreover, this mechanism of self-regulation appears to be ligand-specific, as IRAK-1 mRNA and protein levels are normal in cells following LPS-induced tolerance [54].

Recently, the crystal structure of the DD region of IRAK-4 was resolved to $1.7 \AA$ [56] and represents our first insight into the structural details of a member of the mammalian IRAK family. The crystal structure reveals a six-helical bundle with a prominent loop, which among IRAKs and Pelle, a Drosophilia homologue, is unique to IRAK-4. This unique loop is highly structured, comprised of an 11 amino acid stretch, which may in the future serve as a potential site for the design of inhibitory molecules of therapeutic value.

\section{TRAF FAMILY}

TRAF family members, comprising TRAF1-6, are proposed to be the adaptor molecules linking upstream receptor signals to gene activation. The TRAF proteins are characterized by the presence of a novel TRAF domain at the $\mathrm{C}$ terminus, which consists of a coiled-coil domain, followed by a conserved TRAF-C domain [57]. The TRAF domain plays an important role by mediating self-association and upstream interactions with receptors and other signaling proteins [58]. The N-terminal of most TRAF proteins (TRAF2-6) contains a really interesting new gene finger motif and several zinc finger motif. As a family of proteins, the TRAFs mediate their actions through the activation of transcription factors of the NF-кB and AP-1 family. However, within the family, each TRAF has evolved to mediate distinct signal transduction cascades in a divergent and a convergent manner.

Initially, TRAF6 was thought to be the sole member of this family to be involved in TLR signaling. TRAF6, a ubiquitin ligase, can ubiquitinate itself on Lys-63 (K63)-linked polyubiquitin chains. Ubiquitination targets proteins for proteolytic degradation or activation, depending on the lysine that is modified. For example, polyubiquitination on Lys-48 targets proteins for adenosine $5^{\prime}$-triphosphate-dependent proteolysis by the $26 \mathrm{~S}$ proteasome [59]. In contrast, polyubiquitination on Lys-63 modulates protein function in the absence of degradation. Polyubiquitination of Lys-63 triggers IKK activation through an as-yet undefined mechanism [60]. As TRAF6 is not degraded following ubiquitination, its signaling must be negatively regulated in some manner. It has been shown that the de-ubiquitination enzyme A20 terminates TLR signaling by removing ubiquitin moieties from TRAF6 [61]. A20-deficient macrophages showed enhanced $\mathrm{NF}-\kappa \mathrm{B}$ activity in response to TLR2, TLR4, and TLR9 ligands.

A number of studies have shown that TLR signaling may be regulated by modulation of TRAF6 signaling or use of alternative TRAF family members. For instance, negative regulation of TRAF6 by $\beta$-arrestin has been demonstrated [62]. Specifically, it has been shown that $\beta$-arrestins interact with
TRAF6 in response to LPS and IL-1 $\beta$ stimulation. $\beta$-Arrestins, in addition to their established roles in receptor desensitization and endocytosis, bind to signaling molecules such as the Src family kinases [63] and the E3 ligase Mdm2 [64, 65]. In terms of TLR signaling, it has been shown that $\beta$-arrestins negatively regulate TLR signaling by preventing oligomerization of TRAF6, thereby inhibiting its autoubiquitination and subsequent signaling pathways such as NF- $\mathrm{KB}$ and AP-1 activation [62].

Recently, a number of studies have shown that in addition to TRAF6, activation of distinct TLRs may result in recruitment of alternative TRAF family members, thus adding to the diversity and complexity of the TLR signaling pathway. For example, a recent study has shown that the C-domain of TRAF1 interacts with the TIR domain of Trif; in fact, TRAF1 inhibited Trif and TLR3-mediated activation of NF-кB, IFN-stimulated response element, and IFN- $\beta$ promoter activity [66]. Moreover, it was shown that TRAF1 is cleaved by caspase- 8 generating two fragments, TRAF1(1-163) and TRAF1(164-416), and it is TRAF1(1-163) that is responsible for its inhibitory effects on Trif-mediated signaling. Also, Mal and MyD88 interact with TRAF1, suggesting that TRAF1 may negatively regulate Mal and MyD88 signaling, although this remains to be fully explored [66].

TRAF3 has recently been identified as a new component of TLR signaling, whereby it is recruited along with TRAF6 [67]. Whereas TRAF6 mediates MAPK and IKK, resulting in the activation of NF- $\mathrm{B}$, and AP-1, resulting in the induction of proinflammatory cytokines, TRAF3 is dispensible in this process. However, TRAF3 is crucial for the recruitment of type 1 IFNs and the anti-inflammatory cytokine IL-10 [67]. TRAF3 also interacts directly with Trif and IRAK-1, as well as the IRF3/7 kinases TBK1, IKKi, and putative intracellular viral receptor, protein kinase receptor [67, 68]. Together, these studies reveal that TRAF3 is a critical adaptor molecule for Trif-mediated IRF3 activation and type-1 IFN induction in response to direct TLR activation and direct viral infection.

\section{PERSPECTIVES}

Our ability to respond appropriately to pathogenic challenges is key to our survival, and the TLRs are the first point of contact that we have with invading organisms. As such, the mechanisms regulating the TLR response must be controlled tightly, first to respond appropriately to the pathogenic challenge and second, to prevent excessive activation of the TLR signaling pathway and thus, to control detrimental/deleterious damage to the host system following TLR activation. Intricate mechanisms by which TLR signaling may be regulated are revealed. Protein degradation, sequestration, and compartmentalization are some of the regulatory mechanisms that have evolved. Ultimately, greater understanding of the mechanisms behind TLR signaling will hopefully lead to the development of novel, therapeutic regimes in the treatment of inflammatory diseases and autoimmunity. 
1. Kawai, T., Akira, S. (2006) TLR signaling. Cell Death Differ. 13, 816825 .

2. Zhang, D., Zhang, G., Hayden, M. S., Greenblatt, M. B., Bussey, C., Flavell, R. A., Ghosh, S. (2004) A Toll-like receptor that prevents infection by uropathogenic bacteria. Science 303, 1522-1526.

3. Yarovinsky, F., Zhang, D., Andersen, J. F., Bannenberg, G. L., Serhan, C. N., Hayden, M. S., Hieny, S., Sutterwala, F. S., Flavell, R. A., Ghosh, S., Sher, A. (2005) TLR11 activation of dendritic cells by a protozoan profilin-like protein. Science 308, 1626-1629.

4. Lauw, F. N., Caffrey, D. R., Golenbock, D. T. (2005) Of mice and man: TLR11 (finally) finds profilin. Trends Immunol. 26, 509-511.

5. O'Neill, L. A. (2006) How Toll-like receptors signal: what we know and what we don't know. Curr. Opin. Immunol. 18, 3-9.

6. O'Neill, L. A., Fitzgerald, K. A., Bowie, A. G. (2003) The Toll-IL-1 receptor adaptor family grows to five members. Trends Immunol. 24, $286-290$.

7. Xu, Y., Tao, X., Shen, B., Horng, T., Medzhitov, R., Manley, J. L., Tong, L. (2000) Structural basis for signal transduction by the Toll/interleukin-1 receptor domains. Nature $\mathbf{4 0 8}, 111-115$.

8. Choe, J., Kelker, M. S., Wilson, I. A. (2005) Crystal structure of human Toll-like receptor 3 (TLR3) ectodomain. Science 309, 581-585

9. Bell, J. K., Botos, I., Hall, P. R., Askins, J., Shiloach, J., Segal, D. M., Davies, D. R. (2005) The molecular structure of the Toll-like receptor 3 ligand-binding domain. Proc. Natl. Acad. Sci. USA 102, 10976-10980.

10. Mockenhaupt, F. P., Cramer, J. P., Hamann, L., Stegemann, M. S., Eckert, J., Oh, N. R., Otchwemah, R. N., Dietz, E., Ehrhardt, S., Schroder, N. W., Bienzle, U., Schumann, R. R. (2006) Toll-like receptor (TLR) polymorphisms in African children: common TLR-4 variants predispose to severe malaria. Proc. Natl. Acad. Sci. USA 103, 177-182.

11. Nishiya, T., DeFranco, A. L. (2004) Ligand-regulated chimeric receptor approach reveals distinctive subcellular localization and signaling properties of the Toll-like receptors. J. Biol. Chem. 279, 19008-19017.

12. Funami, K., Matsumoto, M., Oshiumi, H., Akazawa, T., Yamamoto, A., Seya, T. (2004) The cytoplasmic "linker region" in Toll-like receptor 3 controls receptor localization and signaling. Int. Immunol. 16, 11431154 .

13. Matsumoto, M., Funami, K., Tanabe, M., Oshiumi, H., Shingai, M., Seto, Y., Yamamoto, A., Seya, T. (2003) Subcellular localization of Toll-like receptor 3 in human dendritic cells. J. Immunol. 171, 3154-3162.

14. Lee, J., Chuang, T. H., Redecke, V., She, L., Pitha, P. M., Carson, D. A., Raz, E., Cottam, H. B. (2003) Molecular basis for the immunostimulatory activity of guanine nucleoside analogs: activation of Toll-like receptor 7. Proc. Natl. Acad. Sci. USA 100, 6646-6651.

15. Latz, E., Schoenemeyer, A., Visintin, A., Fitzgerald, K. A., Monks, B. G., Knetter, C. F., Lien, E., Nilsen, N. J., Espevik, T., Golenbock, D. T. (2004) TLR9 signals after translocating from the ER to CpG DNA in the lysosome. Nat. Immunol. 5, 190-198.

16. Zhang, H., Tay, P. N., Cao, W., Li, W., Lu, J. (2002) Integrin-nucleated Toll-like receptor (TLR) dimerization reveals subcellular targeting of TLRs and distinct mechanisms of TLR4 activation and signaling. FEBS Lett. 532, 171-176.

17. Nishiya, T., Kajita, E., Miwa, S., Defranco, A. L. (2005) TLR3 and TLR7 are targeted to the same intracellular compartments by distinct regulatory elements. J. Biol. Chem. 280, 37107-37117.

18. Kato, H., Sato, S., Yoneyama, M., Yamamoto, M., Uematsu, S., Matsui, K., Tsujimura, T., Takeda, K., Fujita, T., Takeuchi, O., Akira, S. (2005) Cell type-specific involvement of RIG-I in antiviral response. Immunity 23, $19-28$.

19. Seth, R. B., Sun, L., Chen, Z. J. (2006) Antiviral innate immunity pathways. Cell Res. 16, 141-147.

20. Yoneyama, M., Kikuchi, M., Natsukawa, T., Shinobu, N., Imaizumi, T., Miyagishi, M., Taira, K., Akira, S., Fujita, T. (2004) The RNA helicase RIG-I has an essential function in double-stranded RNA-induced innate antiviral responses. Nat. Immunol. 5, 730-737.

21. Barton, G. M., Kagan, J. C., Medzhitov, R. (2006) Intracellular localization of Toll-like receptor 9 prevents recognition of self DNA but facilitates access to viral DNA. Nat. Immunol. 7, 49-56.

22. Napirei, M., Karsunky, H., Zevnik, B., Stephan, H., Mannherz, H. G., Moroy, T. (2000) Features of systemic lupus erythematosus in DNase1deficient mice. Nat. Genet. 25, 177-181.

23. Yasutomo, K., Horiuchi, T., Kagami, S., Tsukamoto, H., Hashimura, C., Urushihara, M., Kuroda, Y. (2001) Mutation of DNasel in people with systemic lupus erythematosus. Nat. Genet. 28, 313-314.

24. Okabe, Y., Kawane, K., Akira, S., Taniguchi, T., Nagata, S. (2005) Toll-like receptor-independent gene induction program activated by mam-
25. Yoshida, H., Okabe, Y., Kawane, K., Fukuyama, H., Nagata, S. (2005) Lethal anemia caused by interferon- $\beta$ produced in mouse embryos carrying undigested DNA. Nat. Immunol. 6, 49-56.

26. Chuang, T. H., Ulevitch, R. J. (2004) Triad3A, an E3 ubiquitin-protein ligase regulating Toll-like receptors. Nat. Immunol. 5, 495-502.

27. McCartney-Francis, N., Jin, W., Wahl, S. M. (2004) Aberrant Toll receptor expression and endotoxin hypersensitivity in mice lacking a functional TGF- $\beta 1$ signaling pathway. J. Immunol. 172, 3814-3821.

28. Yamamoto, M., Sato, S., Hemmi, H., Uematsu, S., Hoshino, K., Kaisho, T., Takeuchi, O., Takeda, K., Akira, S. (2003) TRAM is specifically involved in the Toll-like receptor 4-mediated MyD88-independent signaling pathway. Nat. Immunol. 4, 1144-1150.

29. Yamamoto, M., Sato, S., Mori, K., Hoshino, K., Takeuchi, O., Takeda, K. Akira, S. (2002) Cutting edge: a novel Toll/IL-1 receptor domain-containing adapter that preferentially activates the IFN- $\beta$ promoter in the Tolllike receptor signaling. J. Immunol. 169, 6668-6672.

30. Fitzgerald, K. A., McWhirter, S. M., Faia, K. L., Rowe, D. C., Latz, E., Golenbock, D. T., Coyle, A. J., Liao, S. M., Maniatis, T. (2003) IKKe and TBK1 are essential components of the IRF3 signaling pathway. Nat. Immunol. 4, 491-496.

31. McWhirter, S. M., Fitzgerald, K. A., Rosains, J., Rowe, D. C., Golenbock, D. T., Maniatis, T. (2004) IFN-regulatory factor 3-dependent gene expression is defective in Tbk1-deficient mouse embryonic fibroblasts. Proc. Natl. Acad. Sci. USA 101, 233-238.

32. Meylan, E., Burns, K., Hofmann, K., Blancheteau, V., Martinon, F., Kelliher, M., Tschopp, J. (2004) RIPl is an essential mediator of Toll-like receptor 3-induced NF-к B activation. Nat. Immunol. 5, 503-507.

33. Cusson-Hermance, N., Khurana, S., Lee, T. H., Fitzgerald, K. A., Kelliher, M. A. (2005) Ripl mediates the Trif-dependent toll-like receptor 3- and 4-induced NF- $\{\kappa\} B$ activation but does not contribute to interferon regulatory factor 3 activation. J. Biol. Chem. 280, 36560-36566.

34. Sato, S., Sugiyama, M., Yamamoto, M., Watanabe, Y., Kawai, T., Takeda, K., Akira, S. (2003) Toll/IL-1 receptor domain-containing adaptor inducing IFN- $\beta$ (TRIF) associates with TNF receptor-associated factor 6 and TANK-binding kinase 1, and activates two distinct transcription factors, NF-к B and IFN-regulatory factor-3, in the Toll-like receptor signaling. J. Immunol. 171, 4304-4310.

35. Janssens, S., Burns, K., Vercammen, E., Tschopp, J., Beyaert, R. (2003) MyD88S, a splice variant of MyD88, differentially modulates NF-кB- and AP-1-dependent gene expression. FEBS Lett. 548, 103-107.

36. Brint, E. K., Xu, D., Liu, H., Dunne, A., McKenzie, A. N., O’Neill, L. A., Liew, F. Y. (2004) ST2 is an inhibitor of interleukin 1 receptor and Toll-like receptor 4 signaling and maintains endotoxin tolerance. Nat. Immunol. 5, 373-379.

37. Wang, T., Chuang, T. H., Ronni, T., Gu, S., Du, Y. C., Cai, H., Sun, H. Q., Yin, H. L., Chen, X. (2006) Flightless I homolog negatively modulates the TLR pathway. J. Immunol. 176, 1355-1362.

38. Mansell, A., Brint, E., Gould, J. A., O'Neill, L. A., Hertzog, P. J. (2004) Mal interacts with tumor necrosis factor receptor-associated factor (TRAF)-6 to mediate NF-кB activation by Toll-like receptor (TLR)-2 and TLR4. J. Biol. Chem. 279, 37227-37230.

39. Jiang, Z., Mak, T. W., Sen, G., Li, X. (2004) Toll-like receptor 3-mediated activation of NF-KB and IRF3 diverges at Toll-IL-1 receptor domaincontaining adapter inducing IFN-B. Proc. Natl. Acad. Sci. USA 101, $3533-3538$

40. Bin, L. H., Xu, L. G., Shu, H. B. (2003) TIRP, a novel Toll/interleukin-1 receptor (TIR) domain-containing adapter protein involved in TIR signaling. J. Biol. Chem. 278, 24526-24532.

41. Ojaniemi, M., Glumoff, V., Harju, K., Liljeroos, M., Vuori, K., Hallman, M. (2003) Phosphatidylinositol 3-kinase is involved in Toll-like receptor 4-mediated cytokine expression in mouse macrophages. Eur. J. Immunol. 33, 597-605.

42. Gray, P., Dunne, A., Brikos, C., Jefferies, C. A., Doyle, S. L., O'Neill, L. A. (2006) MyD88 adapter-like (Mal) is phosphorylated by Bruton's tyrosine kinase during TLR2 and TLR4 signal transduction. J. Biol. Chem. 281, 10489-10495.

43. Mansell, A., Smith, R., Doyle, S. L., Gray, P., Fenner, J. E., Crack, P. J., Nicholson, S. E., Hilton, D. J., O'Neill L. A., Hertzog, P. J. (2006) Suppressor of cytokine signaling 1 negatively regulates Toll-like receptor signaling by mediating Mal degradation. Nat. Immunol. 7, 148-155.

44. Naiki, Y., Michelsen, K. S., Zhang, W., Chen, S., Doherty, T. M., Arditi, M. (2005) Transforming growth factor- $\beta$ differentially inhibits MyD88dependent, but not TRAM- and TRIF-dependent, lipopolysaccharideinduced TLR4 signaling. J. Biol. Chem. 280, 5491-5495.

45. Cao, Z., Henzel, W. J., Gao, X. (1996) IRAK: a kinase associated with the interleukin-1 receptor. Science 271, 1128-1131. 
46. Li, S., Strelow, A., Fontana, E. J., Wesche, H. (2002) IRAK-4: a novel member of the IRAK family with the properties of an IRAK-kinase. Proc. Natl. Acad. Sci. USA 99, 5567-5572.

47. Muzio, M., Ni, J., Feng, P., Dixit, V. M. (1997) IRAK (Pelle) family member IRAK-2 and MyD88 as proximal mediators of IL-1 signaling. Science 278, 1612-1615.

48. Wesche, H., Gao, X., Li, X., Kirschning, C. J., Stark, G. R., Cao, Z. (1999) IRAK-M is a novel member of the Pelle/interleukin- 1 receptor-associated kinase (IRAK) family. J. Biol. Chem. 274, 19403-19410.

49. Janssens, S., Beyaert, R. (2003) Functional diversity and regulation of different interleukin-1 receptor-associated kinase (IRAK) family members. Mol. Cell 11, 293-302.

50. Zhang, G., Ghosh, S. (2002) Negative regulation of Toll-like receptormediated signaling by Tollip. J. Biol. Chem. 277, 7059-7065.

51. Didierlaurent, A., Brissoni, B., Velin, D., Aebi, N., Tardivel, A., Kaslin, E., Sirard, J. C., Angelov, G., Tschopp, J., Burns, K. (2006) Tollip regulates proinflammatory responses to interleukin-1 and lipopolysaccharide. Mol. Cell. Biol. 26, 735-742.

52. Rao, N., Nguyen, S., Ngo, K., Fung-Leung, W. P. (2005) A novel splice variant of interleukin-1 receptor (IL-1R)-associated kinase 1 plays a negative regulatory role in Toll/IL-1R-induced inflammatory signaling. Mol. Cell. Biol. 25, 6521-6532.

53. Hardy, M. P., O'Neill, L. A. (2004) The murine IRAK2 gene encodes four alternatively spliced isoforms, two of which are inhibitory. J. Biol. Chem. 279, 27699-27708.

54. Siedlar, M., Frankenberger, M., Benkhart, E., Espevik, T., Quirling, M., Brand, K., Zembala, M., Ziegler-Heitbrock, L. (2004) Tolerance induced by the lipopeptide Pam3Cys is due to ablation of IL-1R-associated kinase-1. J. Immunol. 173, 2736-2745.

55. Sato, S., Takeuchi, O., Fujita, T., Tomizawa, H., Takeda, K., Akira, S. (2002) A variety of microbial components induce tolerance to lipopolysaccharide by differentially affecting MyD88-dependent and -independent pathways. Int. Immunol. 14, 783-791.

56. Lasker, M. V., Gajjar, M. M., Nair, S. K. (2005) Cutting edge: molecular structure of the IL-1R-associated kinase-4 death domain and its implications for TLR signaling. J. Immunol. 175, 4175-4179.

57. Chung, J. Y., Park, Y. C., Ye, H., Wu, H. (2002) All TRAFs are not created equal: common and distinct molecular mechanisms of TRAFmediated signal transduction. J. Cell Sci. 115, 679-688.
58. Takeuchi, M., Rothe, M., Goeddel, D. V. (1996) Anatomy of TRAF2. Distinct domains for nuclear factor- $\kappa \mathrm{B}$ activation and association with tumor necrosis factor signaling proteins. J. Biol. Chem. 271, 1993519942.

59. Chau, V., Tobias, J. W., Bachmair, A., Marriott, D., Ecker, D. J., Gonda, D. K., Varshavsky, A. (1989) A multiubiquitin chain is confined to specific lysine in a targeted short-lived protein. Science 243, 1576-1583.

60. Wang, C., Deng, L., Hong, M., Akkaraju, G. R., Inoue, J., Chen, Z. J. (2001) TAK1 is a ubiquitin-dependent kinase of MKK and IKK. Nature 412, 346-351.

61. Boone, D. L., Turer, E. E., Lee, E. G., Ahmad, R. C., Wheeler, M. T., Tsui, C., Hurley, P., Chien, M., Chai, S., Hitotsumatsu, O., McNally, E., Pickart, C., Ma, A. (2004) The ubiquitin-modifying enzyme A20 is required for termination of Toll-like receptor responses. Nat. Immunol. 5, 1052-1060.

62. Wang, Y., Tang, Y., Teng, L., Wu, Y., Zhao, X., Pei, G. (2006) Association of $\beta$-arrestin and TRAF6 negatively regulates Toll-like receptor-interleukin 1 receptor signaling. Nat. Immunol. 7, 139-147.

63. Luttrell, L. M., Daaka, Y., Lefkowitz, R. J. (1999) Regulation of tyrosine kinase cascades by G-protein-coupled receptors. Curr. Opin. Cell Biol. 11, 177-183.

64. Shenoy, S. K., McDonald, P. H., Kohout, T. A., Lefkowitz, R. J. (2001) Regulation of receptor fate by ubiquitination of activated $\beta 2$-adrenergic receptor and $\beta$-arrestin. Science 294, 1307-1313.

65. Wang, P., Gao, H., Ni, Y., Wang, B., Wu, Y., Ji, L., Qin, L., Ma, L., Pei, G. (2003) $\beta$-Arrestin 2 functions as a G-protein-coupled receptor-activated regulator of oncoprotein Mdm2. J. Biol. Chem. 278, 6363-6370.

66. Su, X., Li, S., Meng, M., Qian, W., Xie, W., Chen, D., Zhai, Z., Shu, H. B. (2006) TNF receptor-associated factor-1 (TRAF1) negatively regulates Toll/IL-1 receptor domain-containing adaptor inducing IFN- $\beta$ (TRIF)mediated signaling. Eur. J. Immunol. 36, 199-206.

67. Hacker, H., Redecke, V., Blagoev, B., Kratchmarova, I., Hsu, L. C., Wang, G. G., Kamps, M. P., Raz, E., Wagner, H., Hacker, G., Mann, M., Karin, M. (2006) Specificity in Toll-like receptor signaling through distinct effector functions of TRAF3 and TRAF6. Nature 439, 204-207.

68. Oganesyan, G., Saha, S. K., Guo, B., He, J. Q., Shahangian, A., Zarnegar, B., Perry, A., Cheng, G. (2006) Critical role of TRAF3 in the Toll-like receptor-dependent and -independent antiviral response. Nature 439, $208-211$. 